\title{
Resources and Risks of Discussion as an Interactive Method of Organizing the Educational Process
}

\author{
Tatiana T. Sidelnikova, Diana D. Fatykhova \\ Kazan Federal University \\ E-mail: ttsidelnikova@gmail.com,d.fatikhova@gmail.com
}

Received: 21st October 2017 Accepted: 16th November 2017, Published: 31st December 2017

\begin{abstract}
The article analyzes discussion in organization of "interactive education" as process management of mastering knowledge through organization of human interactions and relationships. In this case, learning is a social, collective, rather than an individual process. The discussion, representing a purposeful and orderly exchange of ideas, judgments, opinions in a group, acts as a method, providing interaction in education.

The study purpose is to identify resources and risks of discussion as an interactive method of organizing educational process. Achieving this goal requires consideration of characteristics, functions, types of discussions, analysis of resources and limitations in its use by higher educational institution teachers.

As a methodological research base, work includes following approaches and methods: systemic and structural-functional approaches, sociological, logical and comparative methods. The empirical base included data of observation included and questionnaire data.

As a research result, we came to following conclusions: an interactive education method requires a lot of preparatory work on part of teacher and necessary skills of "conducting" the course of discussion; teacher is required to constantly maintain necessary level of students' involvement in course of discussion, to comply with ethics of disputes, to have willingness to promptly neutralize emerging conflict situations.; a discussion requires teacher to develop a whole range of personal qualities (quick reaction, humor, tolerance, ability to relieve tension that has arisen, etc.)
\end{abstract}

Keywords: Interactive Education, Interactive Methods, Discussion.

\section{Introduction}

The subject education paradigm assuming that student with his motivation, needs, interests is epicenter means not only parity start in building communication between both subjects (teacher-student(s)), not only constant direct-reverse communication on transfer of cognitive (knowledge) component.

The "interactive education", implementing this paradigm, refers to technologies of learning and mastering knowledge and skills through organization of human communications [1, p. 25]. This is a decisive recognition that learning is a social, collective, but not strictly individual process. And it is precisely these characteristics of education that provide targeted development of competencies and qualities that are adequate, both to one's own needs of an individual and to requirements of society of the XXI century. There are following characteristics constantly called among the latter: ability to set and solve a problem; communicative skills; social responsibility; ability to work with information; critical and systemic thinking; creativity and curiosity; focus on self-development [2, p. 56].

Even a cursory glance is enough to see what an important role in achieving these parameters is played by interactivity in learning and such a method of its implementation as a discussion. This article is devoted to this issue.

\section{Methods}

The use of systemic and structural-functional approaches in discussion study contributed to identification of its role in system of subject education paradigm. The comparative method in discussion study made it possible to identify problems and contradictions of this interactive education method. Empirical methods - observation and questioning included made it possible to track the dynamics of this method application.

\section{Results and Discussion}

The psychological and pedagogical studies of domestic and foreign authors pay much attention to study of applying the problem-evaluation interaction in process of group work, - a discussion that involves holistic learning taking into account thoughts, feelings, convictions of each person. This integrity is also conditioned by fact that a person strives for individual growth and autonomy, but remains dependent on other people at same time.

As a working definition, one can take a discussion (Lat. discussio - research, analysis) - as a collective discussion of any issue, problem, comparison of ideas, opinions, proposals, for which each person has his own, individual position.

The discussion purposefulness is not utilitarian task of providing activity during lessons (but, as a prerequisite!) search for new knowledge (clear for each student) - a benchmark for subsequent independent application, development of certain competencies.

The discussion culture of students, including cognitive, 
creative, aesthetic, ethical, communicative competences, reflects ability to speak and listen, explain, characterize phenomena, processes, events, criticize critically, observe dispute ethics, etc.

For example, as discussed above, discussion develops critical and systemic thinking as a quality needed by a person of XXI century. The actualization of critical thinking during educational process leads not only to a more successful understanding of material under study, but also develops such an important social quality as tolerance. Teachers and psychologists are increasingly focusing on formation of such a habit of thinking and cognitive activity, in which "students can take for granted that people disagree on opinions and beliefs, and treat this circumstance not as an annoying human weakness, but as to possibility for knowledge. They will be able to learn from others, even on their objections, discrepancies in perception, different ways of thinking" [3, p. 123-129].

The discussion is different because a person encounters opinions and judgments that are different from his point of view in course of it, which eventually creates a situation of a "conceptual conflict" that forces him to reconsider his views, or to seek additional information to strengthen his position, resulting in fact that his knowledge of the subject is only multiplied [4, 41].

By itself, developing such skills and qualities as creativity and curiosity, critical and systemic thinking, ability to raise and solve problem, to work with information, discussion fulfills an important social mission as well - it develops personal qualities that are inextricably linked with nature of cognition of world and existence in it, i.e. its socializing effect is doubtless.

The numerous studies on using discussion in various types of training indicate that it is inferior in volume to transfer of information in usual lecture, but at the same time it is effective for consolidating material that has been passed, for creative comprehension of subject studied and for forming value orientations. The discussion is ahead of lecture, reading, audiovisual methods, demonstration according to amount of material learned (5\% - in lecture, $50 \%$ - in discussion), second only to practical actions and training of others [5].

The researchers point to following factors as those contributing to successful material learning during discussion:

a) Familiarization of each participant with information that others have (exchange of information) during discussion;

b) Coexistence of different, dissimilar opinions and assumptions about subject under discussion;

c) Encouraging different approaches to same subject or phenomenon;

d) Encouraging participants to seek a group agreement in form of a common opinion or decision [3, p. 123].

In our opinion, the discussion application resources in student audience are also:
- The content ambiguity of material under discussion, its set of problems and even alternative nature actualized by teacher;

- Accessibility to understanding;

- Mastering with elementary knowledge in field of logic and theory of argumentation by students;

- General and polemical culture (and constant external control, as well as, ideally, self-monitoring of observance of polemical "code of honor");

- The very young age of discussion participants, when "dispute creates some sports".

During discussion, teacher's attention is not focused on a student as an individual, but on group of interacting students who activate each other. According to B.Ts. Badmaev, "when using interactive methods, spirit of competition, rivalry, which is manifested when people collectively search for truth, has strongest effect on intellectual activity. In addition, there is such a psychological phenomenon as infection (not imitation, namely, infection), and any thought expressed by a neighbor is capable of involuntarily evoking one's own, analogous or close to what has been said or, on contrary, completely the opposite one" [6, p. 74].

As we have already pointed out, the discussion can be viewed both as a method of interactive learning and as a special technology. A discussion is used in other education formats as a method: social-psychological trainings, educational business games, "learning by doing" technology, case studies, etc. Being a kind of technology, discussion itself includes other methods and techniques: "brainstorming", synectics, inversion, etc.

The discussion types are very diverse. There are free, not controlled by presenter, and guided discussions according to management degree. There is a written discussion (topic discussion with visualization). The discussions with role-based division - (adapted "Method of Six Hats" by Eduard de Bono), roleplaying discussion with function specification of direct participants - ("Organizer" - "Compiler" "Original" "Instigator" "Wrangler") are very common [7, p. 290]. So there is a logical question: how active, with good theoretical development, is discussion used in educational process? Has it become a working tool for teachers? We refer to study results conducted at Kazan Federal University in 2016 - "The Social Wellbeing of Student Youth of the Republic of Tatarstan". One of the questions of this study concerned education innovativeness and suggested among others answers "Yes", "Episodically", "No" to question of prevalence of education using interactive forms (dialog lectures, business games, discussions, etc.) [8, p. 26-27].

The answer "Yes" was given by 202 students $-44.7 \%$; "Episodically" - 160-35, 4\%. The negative answer was given by 49 people $-10.8 \%$. If we summarize the rather vague "Episodic" indicator (once a semester and for one discipline? There was something in first year of study) and "No", then we would get a result that almost coincided with positive statement $(46.2 \%$ and $44.7 \%$, respectively). So there is a question: how should we 
look at it? Is the glass half-full or half-empty? We can reassure ourselves that almost half of the students are immersed in an interactive environment, but, at the same time, other half is outside this environment and is deprived of opportunity to develop above qualities of a human of XXI century using its resources in educational process.

An analysis of answers to question about development of real projects in various fields by students, involving all-round arguments, elucidating all "For" and "Against", fixed implementation of discussion potential to an even lesser degree. Only $28.3 \%$ of respondents gave an affirmative answer about their participation in projects. Such answers as "episodically" and "no-participation" were given by $47.8 \%$ and $14.8 \%$ of respondents, respectively.

We should state that $62.6 \%$ of students who are not involved or are episodically included in work on projects do not acquire competence of creativity, critical thinking, etc. Almost 50\% of students surveyed are taught without use of interactive forms by teachers (dialog lectures, business games, discussions, etc.). According to clarification of reasons for these $50 \%$ carried out in a conversation with teacher by one of authors of this article, we can make a conclusion that more than $70 \%$ of them would like to have a method of discussion in their teaching arsenal, but do not have tools to conduct it. Another interesting fact is that everyone has tried to organize classes in the form of discussion at least once (at least from teachers of sociopolitical and humanitarian disciplines), but has not got desired result.

Two conclusions follow from this. The first one concerns raising qualifications of teaching staff for this form of training organization. But this question is beyond our competence. The second conclusion - in line with this article - suggests at least a short answer to question "What mistakes can a teacher allow during the discussion?"

It seems that they can be combined into three blocks [9, p. 67-72].

1) Regarding discussion topic:

The ill-considered nature of topic itself and its wording, which itself should be problematic, controversial in nature and attract attention, interest. The teacher unjustifiably believes that it is enough to announce that today, for example, a discussion will be held on first issue of practical lesson plan, and it will immediately inflame.

In addition, there is often a lack of agreement on regulations, which not only disciplines participants, but also preserves "tone" of discussion itself. The teacher's own speech is confused and incomplete, sometimes turning discussion into a teaching monologue.

2) The second block deals with attitude to disputing parties - depending on type of discussion - to individual or group opponent and proponent.

In this case, teacher does not know how to clearly formulate thesis, vaguely voiced by student participating in discussion, does not track loss or substitution of a thesis by discussion participants, willingly or unwillingly supports contender, whose position coincides with his own.

3) The third block is more concerned with such forms of discussion, when group participates in it as a whole. In this case, teacher's mistakes are manifested in presence of:

- "Suppresion" of critical judgments; presenter's desire to "comb out" discussion, smooth out acute angles, reconcile disputants ("friendship won"), which only "dilutes" result; ignoring excessive conformity of participants;

- Weak control of collective character of discussion, insufficient activation of passive, or half-active participants;

- An attempt to stand aside from what is happening in audience, take a position of an outside observer, or, conversely, organize, when self-organization is pushed aside by excessive presenter's desire to order procedures;

- Underestimation of importance and necessity of creating a business, creative and, at same time, uninhibited, relaxed atmosphere;

- Weak use for topic development, maintenance of a polemical beginning in discussion of thoughts, arguments, suggestions and remarks that have been made during discussion, especially if they come from groups of opponents;

- Lack of reaction to situations when students pass from discussion topic to individuals, other incorrect methods of conducting polemics [10, 11].

A number of mistakes is primarily of a psychological nature and arises, if teacher underestimates importance of an emotionally positive attitude toward him by students. If he managed to arrange students, to create a favorable psychological climate, then many difficulties related to interpersonal relations will be avoided during discussion.

\section{Conclusions:}

The material considered enables us to conclude that educational discussion is not so often used by teachers in their practice as a resource, an effective interactive method of teaching. This is due to fact that it requires a lot of preparatory work and special skill, consisting in ability to direct discussion and draw inactive participants into discussion process. It is necessary to constantly maintain interest of students in subject of discussion, weight of arguments, observance of ethical norms, overcome resistance of a certain number of students to discussion due to its high intellectual and psychological load, and quickly neutralize collisions that arise.

\section{Summary}

In conclusion, it should be said that discussion plays an important role in achieving goals of interactive education. However, it can be successful only if it is 
based on high professional competence of teacher. Discussion as a method of interactive education and as a special technology requires teacher to develop a whole range of personal qualities (quickness of reaction, humor, tolerance, ability to relieve tension that has arisen, etc.), without which discussion is impossible.

\section{Acknowledgment}

The work is performed according to the Russian Government Program of Competitive Growth of Kazan Federal University.

\section{References}

[1] Sillaste G.G. Interactive Methods of Teaching a Course in Sociology in a Financial-Economic University: Study Guide. P. 1. Sociology Elective Courses / G.G. Sillaste. - M.: Finanacial Academy under the Government of the Russian Federation, 2004. $-118 \mathrm{p}$.

[2] Klarin M.V. Innovations in Education: Metaphors and Models: Analysis of Foreign Experience / M.V. Klarin. - M.: Nauka, 1997. - 223 p.

[3] Johnson D. Methods of Education: Training in Cooperation / D. Johnson, R. Johnson, E. JohnsonHolubek. - St. Petersburg: Economic School and Others, 2001. $-253 \mathrm{p}$.

[4] Badmaev B.Ts. Psychology and Methodology of Accelerated Learning / B.Ts. Badmaev. - M.: Vlados, 1998. - P. 74.

[5] Noskova L.G. On the Effectiveness of Professional Speech Training. // Higher Education in Russia, 2016, No. 3. P. 67-72. 